\title{
Context-Dependent Regulation of Collagen XVII Ectodomain Shedding in Skin
}

\author{
Wataru Nishie, Ken Natsuga, Hiroaki Iwata, Kentaro Izumi, Hideyuki Ujiie, Ellen Toyonaga, Hiroo Hata, Hideki Nakamura, and \\ Hiroshi Shimizu
}

From the Department of Dermatology, Hokkaido University Graduate School of Medicine, Sapporo, Japan

\author{
Accepted for publication \\ January 15, 2015. \\ Address correspondence to \\ Wataru Nishie, M.D., Ph.D., \\ Department of Dermatology, \\ Hokkaido University Graduate \\ School of Medicine, N15W7 \\ Kita-Ku, Sapporo 060-8638, \\ Japan. E-mail: nishie@med. \\ hokudai.ac.jp.
}

\begin{abstract}
Pemphigoid is a common autoimmune blistering disorder in which autoantibodies target transmembrane collagen XVII (COL17), a component of hemidesmosomes in basal keratinocytes. The ectodomain of COL17 can be cleaved from the cell surface within the juxtamembranous extracellular NC16A domain, and, interestingly, certain autoantibodies of pemphigoid patients preferentially react with the shed ectodomain. These findings suggest that COL17 ectodomain shedding generates neoepitopes on the shed form; however, the regulatory mechanism of the shedding in in vivo skin and the pathogenicity of the neoepitope-targeting antibodies still are uncertain. To address these issues, we produced rabbit antibodies specifically reacting with $\mathrm{N}$-terminal cleavage sites of the shed COL17 ectodomain. The antibodies showed that certain amounts of the human COL17 ectodomain are cleaved physiologically at $\mathrm{Gln}^{525}$ in in vivo skin. In contrast, migrating human keratinocytes cleave COL17 at Leu ${ }^{524}$ but not at $\mathrm{Gln}^{525}$. The passive transfer of antibodies reacting with an $\mathrm{N}$-terminal cleavage site of the mouse COL17 ectodomain into neonatal wild-type mice failed to induce blister formation, even though the antibodies bound to the dermal-epidermal junctions, indicating that cleavage site-specific antibodies have reduced or absent pathogenicity for blister formation. This study shows the ectodomain shedding of COL17 to be a physiological event in in vivo human skin that probably generates nonpathologic epitopes on the cleavage sites. (Am J Pathol 2015, 185: 1361-1371; http://dx.doi.org/ 10.1016/j.ajpath.2015.01.012)
\end{abstract}

Bullous pemphigoid (BP), a major pemphigoid subtype, is a common autoimmune blistering skin disease in which autoantibodies (autoAbs) target two hemidesmosomal components: transmembrane collagen XVII (COL17)/BP180 and the plakin family protein BP230. ${ }^{1-5}$ Previous studies have shown that COL17 is the major autoantigen not only for BP but also for other pemphigoid disorders, including linear IgA bullous dermatosis, mucous membrane pemphigoid, pemphigoid gestationis, and lichen planus pemphigoides. ${ }^{3}$ The major epitopes for BP autoAbs tightly cluster within the juxtamembranous noncollagenous NC16A domain of COL17, and the majority of IgG autoAbs react with this region. ${ }^{6,7}$ Passive transfer experiments of rabbit $\operatorname{IgG}^{8}$ and autoAbs from BP patients ${ }^{9}$ into wild-type (WT) or transgenic neonatal mice have shown pathogenic roles of Abs to the NC16A domain in blister formation.

COL17 is a type II-oriented transmembrane glycoprotein whose $\mathrm{N}$-terminus and $\mathrm{C}$-terminus are in the cytoplasm and the extracellular matrix (ECM), respectively. ${ }^{10,11}$ As is true for other transmembrane collagen family members, the extracellular domain of COL17 can be cleaved from the cell surface in vitro by a disintegrin and metalloproteases 9,10 , and $17,{ }^{12,13}$ and, interestingly, the ectodomain shedding occurs within the NC16A domain, which contains major epitopes for BP autoAbs. $^{14,15}$ In addition, autoAbs from linear IgA bullous dermatosis $^{16}$ and certain BP patients ${ }^{17}$ are known to react preferentially with the shed ectodomain of COL17, but do not react with the full-length form of COL17. This unique characteristic also was observed in several monoclonal Abs (mAbs) directing the ectodomain of COL17, ${ }^{11,18-20}$ suggesting that neoepitopes appeared on the shed ectodomain of COL17. Among them, mAb 123, which reacts specifically with the shed COL17 ectodomain, has been shown to induce subepidermal blister formation on unfixed human frozen skin in vitro. ${ }^{18}$

Supported by a Grant-in-Aid for Scientific Research (B) grant 24390274 (W.N.) and by a Challenging Exploratory Research grant 20443955 (W.N.). Disclosures: None declared. 
However, the precise pathomechanism for the generation of neoepitopes after COL17 cleavage has not been elucidated.

Recently, we identified physiological N-terminal cleavage sites of human COL17 within the NC16A domain in vitro: $\mathrm{Asp}^{514}$, $\mathrm{Leu}^{524}, \mathrm{Gln}^{525}$, and $\mathrm{Gly}^{526}{ }^{15}$ In addition, rabbit IgG Abs targeting the cleavage site of $\mathrm{Leu}^{524}$ (Ab HK139) react specifically with the shed COL17 ectodomain, indicating that the cleavage of the molecule actually generates neoepitopes. Furthermore, incubation of cryosections of normal human skin (NHS), neutrophils, and Ab HK139 induced dermalepidermal separation in vitro, suggesting pathogenic roles for the neoepitope-specific Abs in blister formation. ${ }^{15}$ However, the Ab HK139 showed mottled staining at the dermalepidermal junction (DEJ) of NHS; thus, Leu ${ }^{524}$ may be a minor cleavage site in a physiological setting and a different cleavage site (or sites) may be involved in vivo. In addition, it still is uncertain whether neoepitope-specific Abs to COL17 are pathogenic for blister formation in vivo.

Here, we tried to determine whether COL17 is cleaved physiologically and whether the ectodomain shedding of COL17 produces neoepitopes in in vivo skin, and we investigated the pathogenic roles of Abs targeting the neoepitopes at the cleavage sites in blister formation. We found that novel Abs targeting a physiological cleavage site showed that the ectodomain of human COL17 is able to be shed and that the cleaved ectodomain is present at the DEJ even in a physiologically steady setting. In contrast, the cleavage site or sites of COL17 ectodomain are targeted differentially when keratinocytes migrate in cultured keratinocytes as well as in in vivo wound skin. Finally, passive-transfer experiments using Abs targeting a candidate $\mathrm{N}$-terminal physiological cleavage site of the mouse COL17 ectodomain failed to induce blister formation in mice, suggesting that the immune reaction to the neoepitopes on the physiological cleavage sites is not pathogenic.

\section{Materials and Methods}

\section{Cell Culture}

Primary normal human epidermal keratinocytes (NHEKs) isolated from neonatal foreskin (Lonza, Basel, Switzerland) were cultured in keratinocyte growth medium (Lonza). To isolate primary mouse keratinocytes (NMKs), neonatal WT mice on a C57BL/6J background (Clea, Tokyo, Japan) were sacrificed, and whole-skin sections were incubated with $0.25 \%$ trypsin EDTA (Life Technologies, Carlsbad, CA) for 1 hour at $37^{\circ} \mathrm{C}$. After the epidermal layer was scraped and incubated with $10 \%$ fetal calf serum in phosphate-buffered saline (PBS; Life Technologies), primary NMKs were seeded on plastic with Cnt-57 medium supplemented with bovine pituitary extract (Cellntec, Bern, Switzerland). Cells up to the fourth passage were used.

\section{Generation of Abs}

Rabbit polyclonal Abs targeting the N-terminal cleavage site $\mathrm{Gln}^{525}$ of human COL17 (Ab Hu-pyro-Glu) were produced by immunizing two New Zealand White rabbits with peptides corresponding to eight amino acids. ${ }^{15}$ Because N-terminal $\mathrm{Gln}^{525}$ is cyclized physiologically to pyroglutamate (pyro-Glu) in vitro, ${ }^{15}$ the peptides pyro-Glu-Gly-Met-Ala-Pro-Ala-AlaGly-Cys were synthesized chemically using L-pyroglutamic acid (Hokudo, Sapporo, Japan). The peptides were coupled with keyhole limpet hemocyanin through carboxyl terminal Cys with m-maleimidobenzoyl-N-hydroxysuccinimide ester. ${ }^{15}$ Whole IgG from the immunized rabbit serum first was affinity-purified with protein A Sepharose (ProteNova, Takamatsu, Japan) according to the manufacturer's protocol. A portion of the whole IgG Abs were immunoadsorbed using chemically synthesized R7 peptide (Asp ${ }^{522}$ to $\operatorname{Gln}^{545}$ of human COL17; Hokudo) to eliminate Abs that react with the full-length COL17. Briefly, whole IgG Abs were dialyzed to PBS, followed by incubation with R7 peptide-coupled, NHSactivated High Performance (GE Healthcare, Little Chalfont, UK), as previously published. ${ }^{15,21}$ Flow-through samples containing unbound Abs were collected, and the concentration was measured using the BCA protein assay kit (Thermo Fisher Scientific, Rockford, IL). The immunoadsorbed Ab Hu-pyroGlu was used for experiments in this study unless otherwise specified. Rabbit Abs against the NC16A domain $\left(\mathrm{Glu}^{490}\right.$ to $\mathrm{Arg}^{566}$ of human COL17, $\left.\mathrm{Ab} \mathrm{Hu}-\mathrm{NC} 16 \mathrm{~A}\right)^{21}$ targeting the cleavage site $\mathrm{Leu}^{524}$ (Ab HK139) ${ }^{15}$ and the C-terminus $\left(\mathrm{Val}^{1192}\right.$ to Pro ${ }^{1497}$ of human COL17, Ab 09040) ${ }^{22}$ were produced as previously described.

Rabbit polyclonal Abs targeting a candidate N-terminal cleavage site of mouse COL17, Gln ${ }^{532}$ (Ab Mo-pyro-Glu), were produced by immunizing rabbits with the synthetic peptides pyro-Glu-Ala-Glu-Ala-Pro-Ser-Leu-Gly-Cys (Hokudo). The whole Abs first were prepared by using protein A Sepharose as described earlier, and they were affinity-purified further with antigenic peptides by using the SulfoLink Immobilization Kit (Thermo Fisher Scientific) according to the manufacturer's instructions. Precise information about epitopes for these Abs are illustrated in Figure 1. To produce rabbit Abs against the mouse NC14A domain of COL17 (Ab MoNC14A), glutathione $S$-transferase (GST)-conjugated mouse NC14A protein (GST-NC14A) was prepared as previously described. ${ }^{9}$ Briefly, PCR products encoding $75-$ amino acid NC14A polypeptides (from $\mathrm{Glu}^{499}$ to $\mathrm{Arg}^{573}$ of mouse COL17) were amplified using the primers mNC14A-F (forward, 5'-CCGAATTCGAGGAGGTAAGAAAGCTGAA- ${ }^{\prime}$; the EcoRI restriction site is underlined) and mNC14A-R (reverse, 5'-GGGCGGCCGCTCTGAGATTCCCGTTCTCCT- $3^{\prime}$; the NotI restriction site is underlined), and cDNA from mouse keratinocytes. The 243-bp PCR product was digested by EcoRI and NotI, followed by integration into the pGEX-6P-1 vector (GE Healthcare). After DNA sequences were confirmed, the expression vector was transformed into Escherichia coli (BL21; GE Healthcare). The cells were lysed by B-PER protein extraction reagent (Thermo Fisher Scientific), and GSTNC14A protein was purified by GSTrap affinity column (GE Healthcare) according to the manufacturer's 


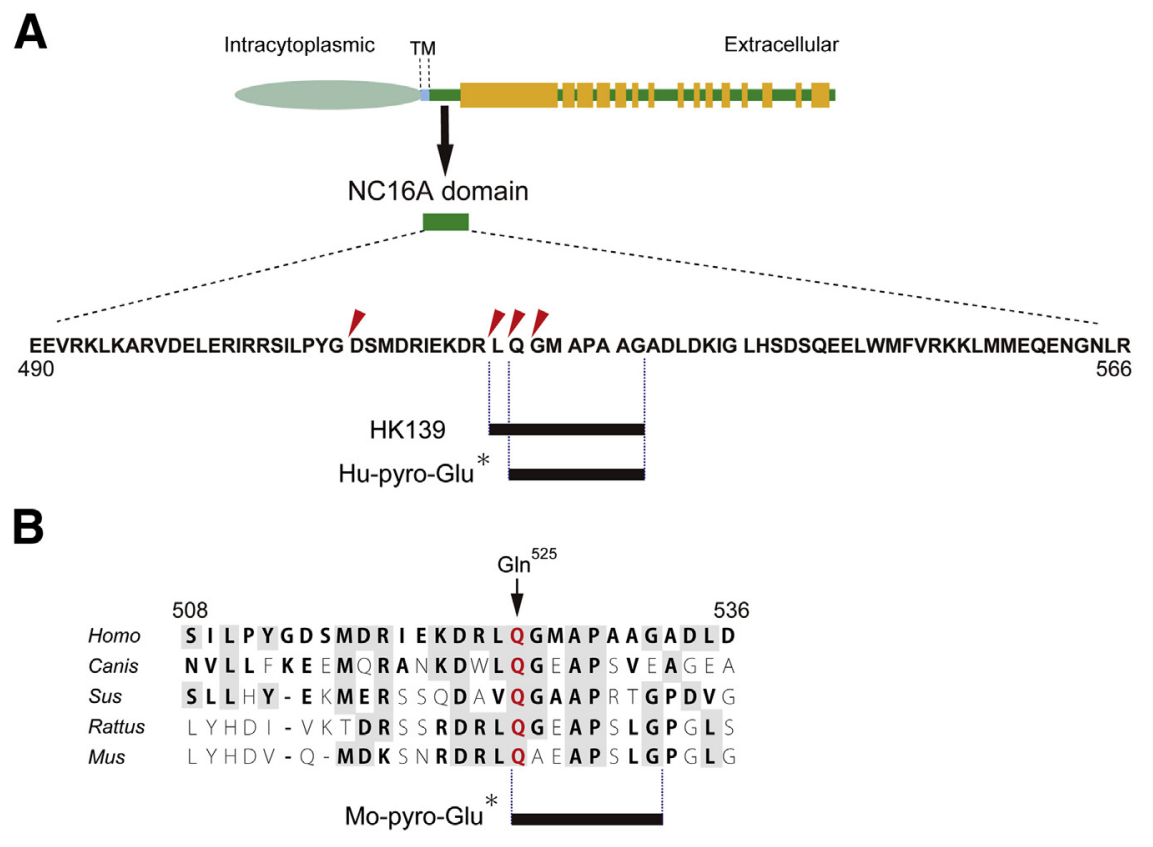

Figure 1 The physiological $\mathrm{N}$-terminal cleavage site $\mathrm{Gln}^{525}$ of human collagen XVII (COL17) is highly conserved among different species. A: Black arrow and dashed lines illustrate amino acid sequences of juxtamembraneous NC16A domain of COL17. Physiologically, the ectodomain of human COL17 is cleaved at different sites within the NC16A domain, including at Asp ${ }^{514}, \mathrm{Leu}^{524}, \mathrm{Gl}^{525}$, and $\mathrm{Gly}^{526}$ (red arrowheads). Epitopes of antibodies (Abs) HK139 and Hu-pyro-Glu are shown. The N-terminal $\mathrm{Gln}^{525}$ of the immunized peptides for the Ab Hu-pyro-Glu has been changed chemically into pyro-Glu (asterisk). B: The N-terminal cleavage site $\mathrm{Gln}^{525}$ for human COL17 (black arrow indicating red letters) is highly conserved among different species, suggesting that $\mathrm{Gln}^{525}$ is a vital amino acid. The corresponding epitope of immunized peptides to produce the Ab Mo-pyro-Glu is shown (black bar). The N-terminal Gln of the immunized peptides for the Ab also was changed chemically into pyro-Gln (asterisk). TM, transmembrane.

instructions. The correct expression of GST-NC14A as a GST-fusion protein was confirmed by digesting it with PreScission Protease (GE Healthcare). Similarly, recombinant mouse NC14A protein with C-terminal hexahistidine and herpes simplex virus tag sequences (NC14A-His $\cdot$ herpes simplex virus) was produced using the same primers and the pET-44a (+) vector (Novagen, Madison, WI). For the control, an empty vector was used. To obtain the $\mathrm{Ab}$ Mo-NC14A, four New Zealand White rabbits were immunized with the purified GST-NC14A protein, and the whole IgG was affinity-purified as described earlier. The specificity of the Ab Mo-NC14A to the NC14A domain of mouse COL17 was assessed by immunoblotting using NC14AHis $\bullet$ herpes simplex virus protein.

\section{Immunoblotting and Immunohistochemistry}

For immunoblotting, subconfluent NHEKs or NMKs were lysed for 30 minutes on ice in a buffer of $1 \%$ Nonidet P-40, 0.1 $\mathrm{mol} / \mathrm{L} \mathrm{NaCl}, 25 \mathrm{mmol} / \mathrm{L}$ Tris-HCl, $\mathrm{pH}$ 7.4, $10 \mathrm{mmol} / \mathrm{L}$ EDTA, and $1 \mathrm{mmol} / \mathrm{L}$ Pefabloc SC (Sigma, St. Louis, MO). ${ }^{12}$ Medium proteins were precipitated by ethanol. ${ }^{12}$ To address spontaneous cyclization of N-terminal $\operatorname{Gln}^{525}$ into pyro-Glu in the human COL17 ectodomain, a sterilized culture medium of NHEKs was incubated at $37^{\circ} \mathrm{C}$ for 2,4 , or 9 days before ethanol precipitation. The ECM proteins were prepared as described elsewhere. ${ }^{23,24}$ Briefly, the cell layer was incubated at room temperature with $20 \mathrm{mmol} / \mathrm{L} \mathrm{NH} \mathrm{NH}_{4} \mathrm{OH}$ solution after being washed with PBS. After all of the cells had detached, the ECM was washed thoroughly with PBS and dissolved in SDS-polyacrylamide gel electrophoresis sample buffer. The samples were separated on SDS-polyacrylamide gel electrophoresis $7 \%$ polyacrylamide gels, followed by transfer onto a nitrocellulose membrane. The following Abs were used: anti-herpes simplex virus-tag Ab (A00624; Genscript, Piscataway, $\mathrm{NJ}$ ), the rabbit polyclonal Abs described earlier, and mouse mAb NC16A-3 directing the NC16A domain of human $\mathrm{COL} 17^{20}$ (provided by Prof. Leena Bruckner-Tuderman, Freiburg Institute for Advanced Studies, Freiburg im Breisgau, Baden-Württemberg, Germany). After incubation with a horseradish peroxidase-conjugated secondary $\mathrm{Ab}$, signals were visualized by ECL-plus (GE Healthcare). For indirect immunofluorescence (IIF) microscopy, skin specimens of NHS, skin from a COL17-lacking epidermolysis bullosa patient, ${ }^{25}$ normal mouse skin (NMS), mouse Col17-lacking mice skin, ${ }^{9}$ and NHEKs cultured on coverslips for 18 hours were used. For IIF of wounded skin, three skin ulcer samples taken after surgery from a 26-year-old man, a 68-year-old man, and a 69-year-old man were used (one from each individual), from whom pilomatricoma on the left upper arm, the left inguinal lymph nodes, and malignant melanoma on the left sole had been removed surgically 25,37 , and 35 days before, respectively. For some experiments, human or mouse skin sections were incubated with $1 \mathrm{~mol} / \mathrm{L} \mathrm{NaCl}$ to produce artificial blisters for 14 and 24 hours at $4^{\circ} \mathrm{C}$, respectively. The skin specimens were mounted and snap-frozen in optimal cutting temperature compound (Tissue-Tek O.C.T.; Sakura, Alphen aan den Rijn, the Netherlands), and 5- $\mu \mathrm{m}$ cryosections were incubated with antiserum, purified rabbit polyclonal IgG Abs, or serum from rabbit $\mathrm{Ab}$-injected mice for 1 hour at room temperature and detected with 1:1000 diluted Alexa488conjugated anti-rabbit IgG (Life Technologies) and propidium iodide (Wako, Osaka, Japan). For direct immunofluorescence microscopy, Alexa488-conjugated anti-rabbit IgG and fluorescein isothiocyanate-conjugated anti-mouse C3 (MP Biomedicals, Solon, $\mathrm{OH}$ ) were used. IIF on cultured NHEKs was performed using rhodamine phalloidin (Life Technologies) as previously described, ${ }^{24}$ and images were observed by 

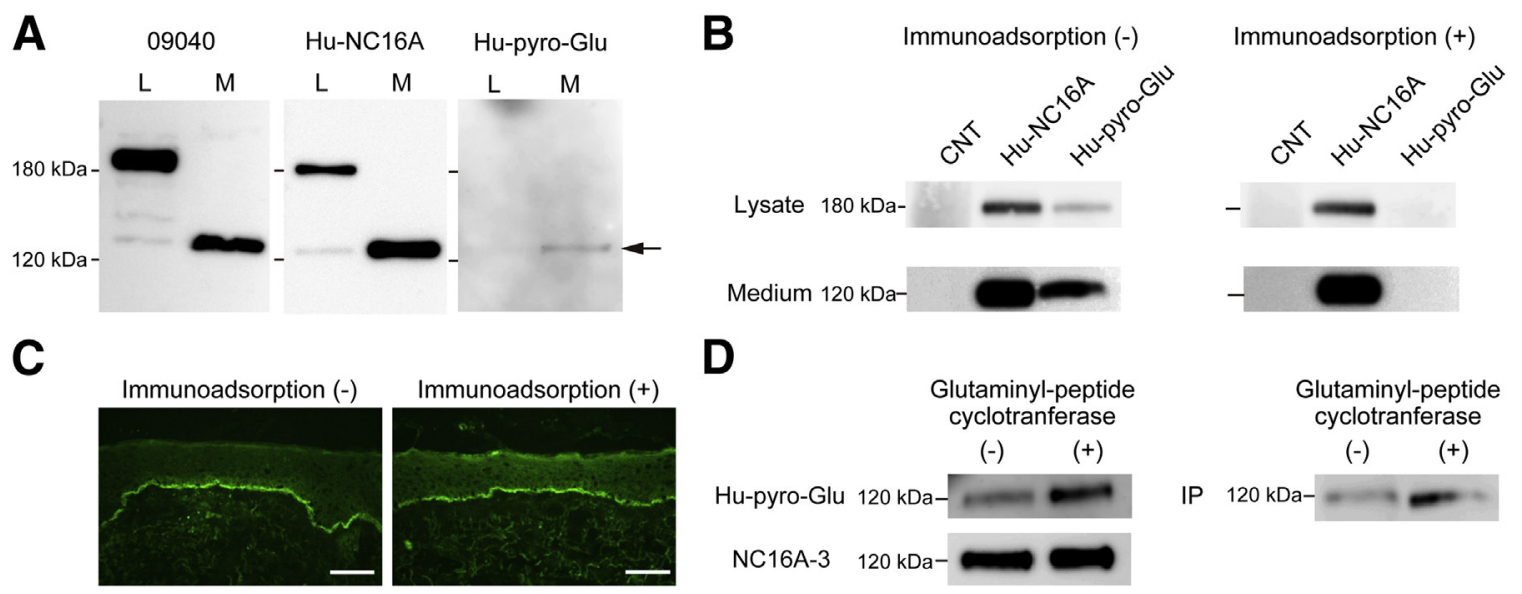

D
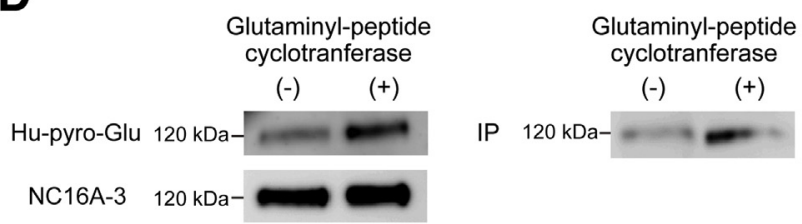

E

$\mathbf{F}$ Incubation time (day)
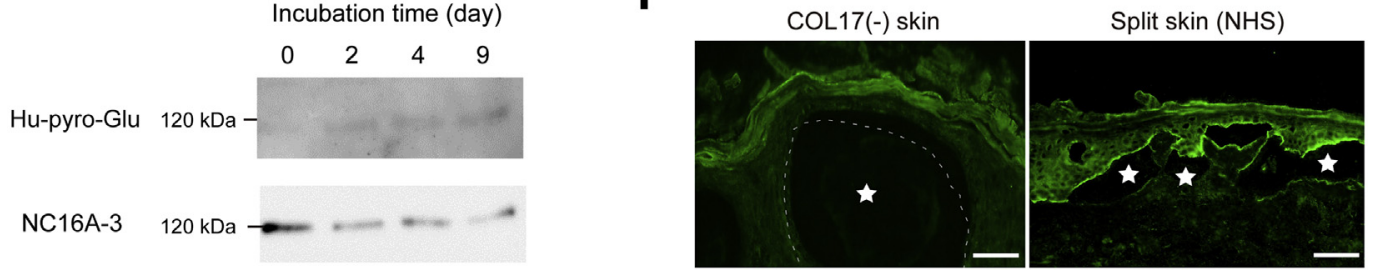

Figure 2 Ectodomain shedding of human collagen XVII (COL17) in cultured primary normal human epidermal keratinocytes (NHEKs) and in in vivo human skin. A: Western blot using cell lysate $(\mathrm{L})$ and culture medium protein $(\mathrm{M})$ from NHEKs shows that the antibody (Ab) Hu-pyro-Glu weakly reacts only with the $120-\mathrm{kDa}$ shed ectodomain (arrow) and not with the 180-kDa full-length form of COL17. In contrast, Abs 09040 and Hu-NC16A, which target the C-terminus and the NC16A domain, respectively, react with both the full-length and the shed forms. The Ab Hu-NC16A reacts more strongly with the 120-kDa shed ectodomain than with the full-length form. B: Immunoprecipitation using Abs Hu-NC16A and Hu-pyro-Glu before and after immunoadsorption using synthesized R7 peptide spanning $\mathrm{Asp}^{522}$ to $\mathrm{Gln}^{545}$. Immunoprecipitation samples from cell lysate and culture medium of NHEKs were detected by $\mathrm{Ab} 09040$ and monoclonal $\mathrm{Ab}$ NC16A-3, respectively. The Ab Hu-pyro-Glu before immune adsorption weakly reacts with full-length COL17, whose reactivity was abolished after immunoadsorption of the Abs. The reactivity of the Ab Hu-pyro-Glu to the shed ectodomain also was abolished after the immunoadsorption. C: Immunoreactivity of the Ab Hu-pyro-Glu on normal human skin does not differ before versus after immunoadsorption (Ab concentration, $0.005 \mu \mathrm{g}$ each). D: Treatment of NHEK culture medium with recombinant human glutaminyl-peptide cyclotransferase. Western blot shows an increase in the 120-kDa COL17 ectodomain detected by $\mathrm{Ab}$ Hu-pyro-Glu in medium protein treated with glutaminyl-peptide cyclotransferase, the reactivity by monoclonal Ab NC16A-3 is unchanged. The Ab Hu-pyroGlu can immunoprecipitate small amounts of COL17 ectodomain from nontreated culture medium $(3 \mathrm{~mL})$ of NHEKs; in contrast, the Ab immunoprecipitates increase 120-kDa COL17 ectodomain from the glutaminyl-peptide cyclotransferase-treated medium protein; detected by monoclonal Ab NC16A-3. E: Spontaneous cyclization of N-terminal $\mathrm{Gln}^{525}$ of the shed COL17 ectodomain into pyro-Glu. Long-term incubation of NHEK culture medium at $37^{\circ} \mathrm{C}$ produces increased amounts of COL17 ectodomain, which is detectable by immunoadsorbed Ab Hu-pyro-Glu of COL17 in a time-dependent manner. In contrast, reactivity of the 120-kDa Ab NC16A-3 is decreased, indicating that certain amounts of COL17 ectodomain are degraded during incubation (a stripped membrane was used). F: The Ab Hu-pyro-Glu fails to react with skin sections obtained from a COL17-lacking epidermolysis bullosa patient. The Ab reacts with the epidermal side of artificial blisters (stars) of NHS induced by pre-incubation with $1 \mathrm{~mol} / \mathrm{L} \mathrm{NaCl}$ for 14 hours. Scale bars: $50 \mu \mathrm{m}(\mathbf{C}$ and $\mathbf{F}$ ). IP, immunoprecipitation.

fluorescence microscopy (IX-51; Olympus, Tokyo, Japan) or confocal microscope (FX81, Olympus).

\section{Immunoprecipitation}

Immunoprecipitation was performed using rabbit polyclonal Abs as described earlier, normal rabbit polyclonal IgG (37415; Abcam, Cambridge, UK), cell lysates, or medium of cultured NHEKs and NMKs. Briefly, $50 \mu \mathrm{L}$ of protein-G Sepharose (GE Healthcare) was coupled with $50 \mu \mathrm{g}$ of Abs Hu-pyro-Glu, Hu-NC16A, or normal rabbit IgG, followed by incubation with cell lysates of NHEKs or $1 \mathrm{~mL}$ of culture medium for 1 hour at room temperature. After extensive washing of the beads with PBS containing $0.1 \%$ NP-40, bound proteins were eluted by boiling for 5 minutes with $1 \times$ SDS sample buffer. To assess whether the N-terminus of the shed COL17 ectodomain was cyclized from
Gln ${ }^{525}$ into pyro-Glu, $3 \mathrm{~mL}$ of NHEK culture medium was concentrated and buffer was exchanged to $25 \mathrm{mmol} / \mathrm{L}$ HEPES, pH 7.0, using an Amicon Ultra Centrifuge Filter Unit (30 kDa; Millipore, Darmstadt, Germany). The concentrated culture medium was treated with $1 \mu \mathrm{g}$ of recombinant human glutaminyl-peptide cyclotransferase $(6368-\mathrm{ZN}$; $\mathrm{R} \& \mathrm{D}$ Systems, Minneapolis, MN) for 24 hours at $37^{\circ} \mathrm{C}$ followed by immunoprecipitation using $\mathrm{Ab} \mathrm{Hu}-$ pyro-Glu. TruBlot (Rockland Immunochemicals, Limerick, PA) was used as a secondary Ab.

\section{Passive Transfer of the Cleavage-Specific Abs into Mice}

Neonatal and 8-week-old WT C57BL/6J mice were used. The neonatal mice received a single intraperitoneal injection of $250 \mu \mathrm{g} / \mathrm{g} / \mathrm{body}$ weight of the Ab Mo-pyro-Glu $(n=6)$, which had been affinity-purified with peptide beads, or 


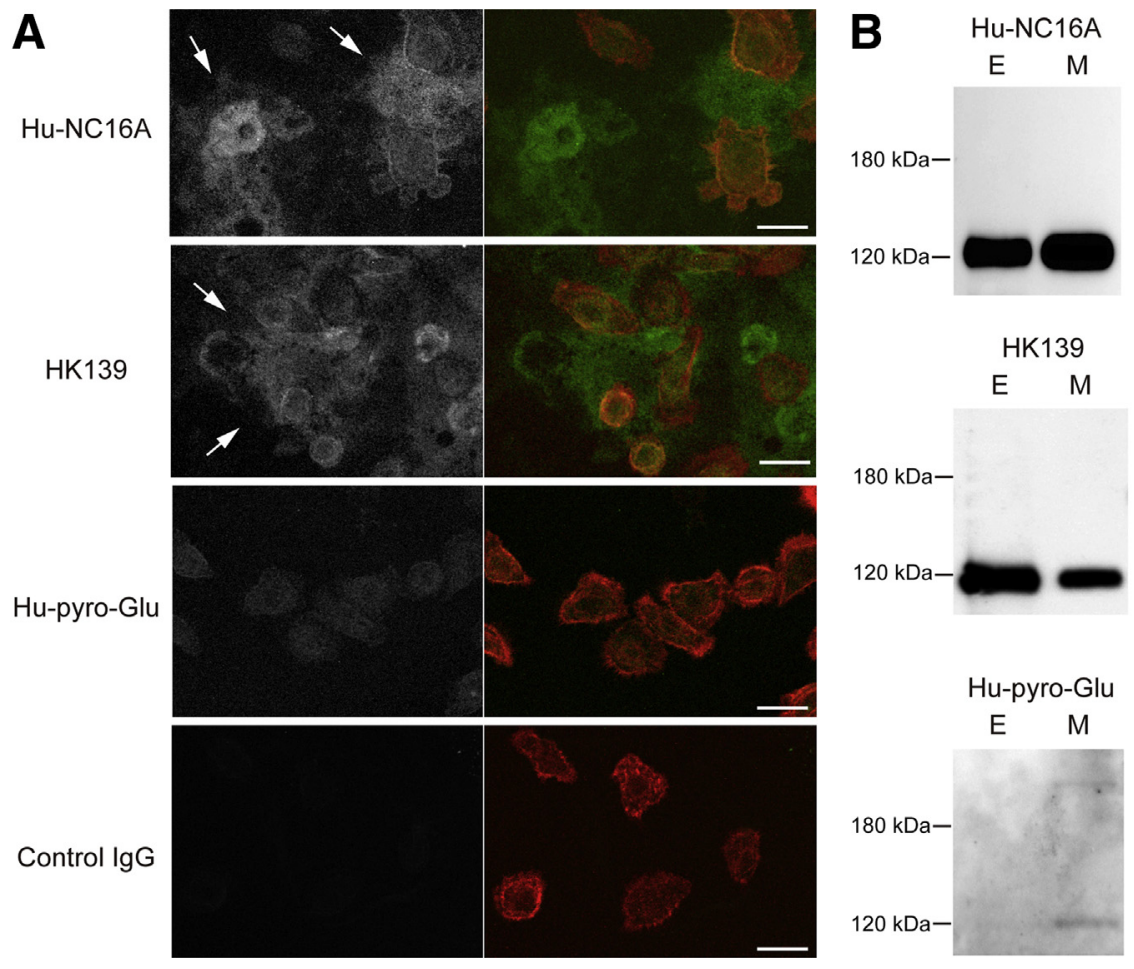

Figure 3 Ectodomain shedding of collagen XVII (COL17) in migrating keratinocytes. A: The cleaved ectodomain of COL17 in the extracellular matrix (arrows in left column, green in right column) of the primary normal human epidermal keratinocytes is detectable by both the antibodies (Abs) targeting the NC16A domain (Ab Hu-NC16A) and the cleavage site of Leu ${ }^{524}$ (Ab HK139), whereas not detectable by the Ab Hu-pyro-Glu and normal rabbit IgG as control. Red: Rhodamine phalloidin. B: Western blot using extracellular matrix samples (E) and culture medium protein (M) of normal human epidermal keratinocytes shows detection of the $120-\mathrm{kDa}$ cleaved ectodomain of COL17 in the extracellular matrix by Ab Hu-NC16A and the Ab HK139 but no detection by Ab Hu-pyroGlu. Scale bars: $25 \mu \mathrm{m}(\mathbf{A})$.
$2000 \mu \mathrm{g} / \mathrm{g} / \mathrm{body}$ weight of whole $\mathrm{IgG}$ containing the $\mathrm{Ab}$ Mo-NC14A $(n=4)$. Forty-eight hours after the injection, back skin was evaluated separately by two investigators for detachment by gentle rubbing. ${ }^{9,21}$ For adult mice, $500 \mu \mathrm{L}$ of antiserum containing the Ab Mo-pyro-Glu was injected subcutaneously each day for 5 consecutive days, and skin disease was assessed 3 days after the last injection $(n=4)$. For the control, preimmune serum or normal whole-rabbit $\operatorname{IgG}$ (Abcam) was used.

All mouse procedures were approved by the Institutional Animal Care and Use Committee of Hokkaido University. This study was approved by the Ethics Committee of Hokkaido University, and fully informed consent was obtained from all patients and healthy volunteers for the use of their materials.

\section{Results}

Gln ${ }^{525}$ of COL17 Is a Highly Conserved, Physiological, $\mathrm{N}$-Terminal Cleavage Site in Normal Human Skin

We previously reported that different cleavage sites cluster within the NC16A domain of COL17 (Figure 1A). ${ }^{15}$ Although amino acid sequences covering the NC16A domain show significant differences among different species, ${ }^{10,26}$ the cleavage site $\mathrm{Gln}^{525}$ is highly conserved (Figure 1B), indicating that it is a functionally important amino acid. Interestingly, our previous studies showed that the $\mathrm{N}$-terminal $\mathrm{Gln}^{525}$ is unique because its $\mathrm{N}$-terminus is cyclized naturally by changing from Gln into pyro-Glu after cleavage in vitro. ${ }^{15}$ To assess whether human COL17 actually is cleaved at this site and whether the N-terminus was changed from $\mathrm{Gln}^{525}$ to pyro-Glu ${ }^{525}$ in vivo, we produced rabbit Abs (Ab Hu-pyro-Glu) targeting the modified cleavage site (Figure 1A). Western blot showed that the $\mathrm{Ab}$ Hu-pyro-Glu reacts weakly with the $120-\mathrm{kDa}$ shed ectodomain and does not react with the 180-kDa full-length form of COL17 (Figure 2A). However, immunoprecipitation studies using high doses of the Ab and plenty of NHEK cell lysate showed that the $\mathrm{Ab}$ was able to immunoprecipitate small amounts of full-length COL17 (Figure 2B). Therefore, we immunoadsorbed the Ab using synthesized R7 peptide $\left(\mathrm{Asp}^{522}\right.$ to $\mathrm{Gln}^{545}$ ), ${ }^{21}$ which does not contain $\mathrm{Gln}^{525}$ as an $\mathrm{N}$-terminal cleavage site, and the treatment eliminated the reaction to full-length COL17 (Figure 2B). The ability of Ab Hu-pyro-Glu to immunoprecipitate the 120-kDa COL17 ectodomain also was abolished after immunoadsorption (Figure 2B), indicating that the shed COL17 ectodomain with pyro-Glu N-terminus was not present or was present only in small amounts in the NHEK culture medium. Immunoreactivity of the Ab Hu-pyro-Glu to NHS was not reduced after immunoadsorption (Figure 2C); thus, we speculated that N-terminal cyclization from $\mathrm{Gln}^{525}$ into pyro-Glu of the shed COL17 ectodomain may occur preferentially in vivo and that the cleaved molecule in the NHEK culture medium had noncyclized N-terminal Gln ${ }^{525}$. To address these hypotheses, we treated a culture medium of NHEKs with recombinant human glutaminyl-peptide cyclotransferase, an enzyme that cyclizes N-terminal Gln into pyro-Glu. ${ }^{27}$ After the treatment, Ab Hu-pyro-Glu showed increased reaction to the shed COL17 ectodomain (Figure 2D), indicating that the shed COL17 ectodomain 

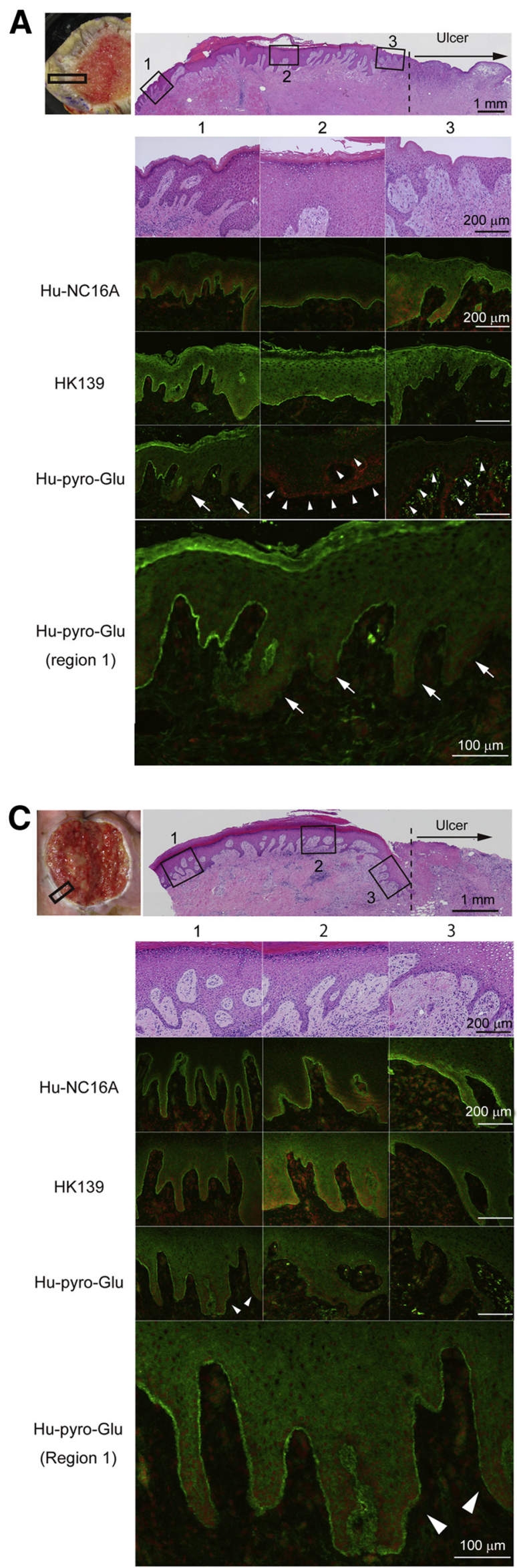

B

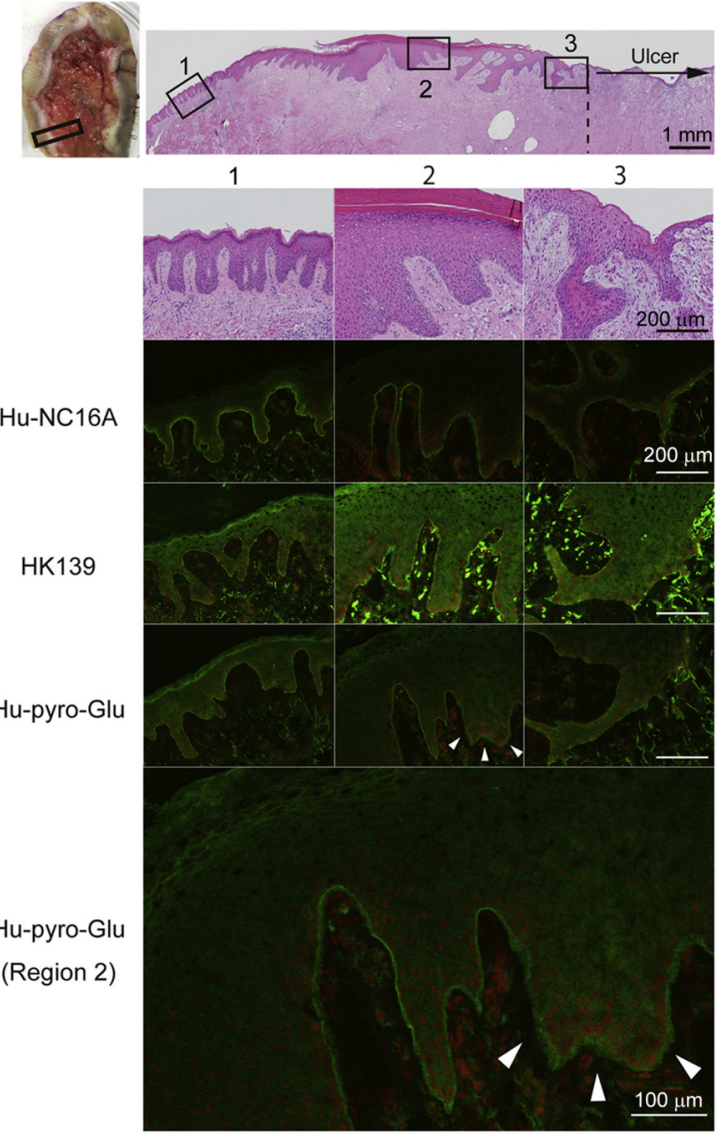

Figure 4 Ectodomain shedding of collagen XVII (COL17) in ulcers after surgery. A: Ulcers after surgery from case 1 (a 26-year-old man, 25 days after the surgery). In contrast to the antibodies (Abs) Hu-NC16A and HK139, signals at the dermal-epidermal junction (DEJ) by the Ab Hu-pyro-Glu are diminished at the leading edge and at the re-epithelialized hypertrophic epidermis (regions 3 and 2, respectively; arrowheads). Note the gradual reduction of Ab Hu-pyro-Glu staining at the DEJ in the transitional region from normal to hypertrophic skin (region 1, arrows). The lowest panel is a high magnification of the Ab-Hu-pyro-Glu in region 1. B: Case 2 (a 68-year-old man, 37 days after the surgery). Signals at the DEJ by the Ab Hu-pyro-Glu are diminished at the leading-edge epidermis (region 3). The lowest panel is a high magnification of the Ab-Hu-pyro-Glu in region 2, in which there is a gradual reduction of the Ab Hu-pyro-Glu staining at the DEJ in the transitional hypertrophic skin (arrowheads). In case 2, the Ab Hu-NC16A shows expression patterns similar to those of the Ab Hu-pyro-Glu. C: Case 3 (a 69-year-old man, 35 days after the surgery). Note the gradual reduction of the Ab Hu-pyro-Glu staining at the DEJ in the transitional region from normal to hypertrophic skin (region 1, arrowheads). The lowest panel is a high magnification of the Ab-Hu-pyro-Glu in region 1 . Dermal staining by Abs HK139 in regions 2 and 3 in case 2, and by Ab Hu-pyro-Glu in region 3 in both cases is attributed to crossreactivity to unknown dermal products that probably are associated with inflammation. Note that the normal skin region is lacking in case $3(\mathbf{C})$. Green represents Abs; red represents propidium iodide. 
with noncyclized N-terminal $\mathrm{Gln}^{525}$ is present in the NHEK culture medium. In addition, the Ab Hu-pyro-Glu precipitated increased amounts of COL17 ectodomain from the NHEK culture medium treated with glutaminyl-peptide cyclotransferase (Figure 2D), proving specific binding of the $\mathrm{Ab}$ to the cyclized pyro-Glu N-terminus of the shed molecule. Furthermore, spontaneous cyclization of $\mathrm{N}$-terminal $\mathrm{Gln}^{525}$ in culture medium after incubation at $37^{\circ} \mathrm{C}$ was confirmed (Figure $2 \mathrm{E}$ ). These findings prove that a majority of the COL17 ectodomain in the NHEK culture medium has a noncyclized $\mathrm{Gln}^{525} \mathrm{~N}$-terminus and that the $\mathrm{Ab} \mathrm{Hu}$-pyro-Glu reacts specifically with the pyro-Glu $\mathrm{N}$-terminal shed COL17 ectodomain. The immunoadsorbed Ab Hu-pyro-Glu failed to react with skin sections obtained from a patient with epidermolysis bullosa but lacking COL17 (Figure 2F). This confirmed that the reaction was specific to human COL17. Interestingly, the Ab Hu-pyro-Glu showed positive staining on the epidermal side of artificial blisters of NHS induced by pre-incubation with $1 \mathrm{~mol} / \mathrm{L} \mathrm{NaCl}$ for 14 hours (Figure 2F), suggesting the presence of an unknown binding molecule or molecules that anchor the shed COL17 ectodomain in the ECM. The reactivity of the Abs to the epidermal side was diminished when the incubation time of NHS with $1 \mathrm{~mol} / \mathrm{L} \mathrm{NaCl}$ was increased to 24 hours to induce artificial blisters (not shown), indicating that epitopes of the $\mathrm{Ab}$ Hu-pyro-Glu probably are cleaved off by internal proteases.

\section{COL17 Ectodomain Shedding in Migrating Keratinocytes Is Regulated Differentially in Vitro Versus in Vivo}

We previously showed that migrating NHEKs cleave COL17 at Leu $^{524}$ and that the cleaved ectodomain of COL17 is detectable by the Ab HK139 targeting this cleavage site as migration tracks in the ECM in vitro. ${ }^{15}$ However, the Ab Hu-pyro-Glu showed no signals in the ECM of cultured NHEKs, whereas both the $A b$ to the NC16A domain (Ab Hu-NC16A) and the Ab HK139 were able to detect large amounts of COL17 as migration tracks (Figure 3A). Consistent with the IIF findings, Western blot using ECM samples of NHEKs showed that the $\mathrm{Ab} \mathrm{Hu}$ pyro-Glu was unable to detect the $120-\mathrm{kDa}$ ectodomain of COL17, whereas both the Ab Hu-NC16A and the Ab HK139 were able to detect that ectodomain (Figure 3B). These results suggest that migrating NHEKs cleave COL17 at sites different from those of the normal steady state in vitro. To address this further in an in vivo setting, we tested the reactivity of the Abs on ulcerated wound skin after surgery as described in Material and Methods. It was found that physiological $\mathrm{N}$-terminal cleavage at $\mathrm{Gln}^{525}$, detectable by the Ab Hu-pyro-Glu, was diminished at the DEJ of the leading-edge epidermis, although slightly reduced but distinct expression of COL17 was detectable by the Ab Hu-NC16A (Figure 4). These results suggest that the ectodomain shedding of COL17 also is regulated differentially in in vivo human wound skin. In addition, different expression patterns were observed in a region intermediate between the re-epithelializing edge and normal skin among different samples. The expression of the $\mathrm{Ab} \mathrm{Hu}-$ pyro-Glu was much weaker in cases 1 and 3 than in case 2 (Figure 4, A-C). In case 2, the Ab Hu-NC16A showed expression patterns similar to those of the Ab Hu-pyro-Glu (Figure 4B). These differences may reflect the difference in time elapsed after surgery (case 1, 25 days; case 2, 37 days; and case 3,35 days).

\section{Passive Transfer of Abs to Neoepitopes on Candidate Physiological Cleavage Sites of Mouse COL17 Ectodomain Fails to Reproduce Blistering Disease in Mice}

The final question was whether Abs to neoepitopes on the cleavage sites of the shed ectodomain of COL17 are pathogenic for blister formation. Because the cleavage site-specific $\mathrm{Ab}$ Hu-pyro-Glu did not react with the COL17-humanized mice skin (not shown), we generated another Ab targeting a candidate physiological cleavage site of mouse COL17. Based on the facts that $\mathrm{Gln}^{525}$ of human COL17 is highly conserved among different species (Figure 1B), we hypothesized that the amino acid $\mathrm{Gln}^{532}$ of mouse COL17, corresponding to the $\mathrm{Gln}^{525}$ of human COL17, may be a major cleavage site of mouse skin in vivo. In addition, we speculated that the $\mathrm{N}$-terminal $\mathrm{Gl}^{532}$ may be modified by changing into pyro-Glu, as seen for the human N-terminal $\mathrm{Gln}^{525}$ of COL17. To address these hypotheses, we produced another rabbit Ab, Mo-pyro-Glu, which targets the candidate $\mathrm{N}$-terminal cleavage site of mouse COL17, whose $\mathrm{Gln}^{532}$ has been changed into pyro-Glu (Figure 1B). The Ab Mo-pyro-Glu reacted strongly with the DEJ of the WT mouse skin, although it failed to react with the COL17-lacking mouse $\left(\mathrm{Coll7}^{-1-}\right)$ skin (Figure 5A), supporting the idea of specificity of the $\mathrm{Ab}$ to mouse COL17. In addition, similar to the Ab Hu-pyro-Glu, the Ab Mo-pyro-Glu reacted with the epidermal side of artificial blisters of NMS pre-incubated with 1 $\mathrm{mol} / \mathrm{L} \mathrm{NaCl}$ for 14 hours (Figure $5 \mathrm{~A}$ ), although its reactivity was lost on skin that had been incubated with $1 \mathrm{~mol} / \mathrm{L} \mathrm{NaCl}$ for 24 hours as observed by Ab Hu-pyro-Glu (not shown). The Ab Mo-pyro-Glu immunoprecipitates the shed mouse COL17 ectodomain (Figure 5B), and Western blotting showed that the $\mathrm{Ab}$ Mo-pyro-Glu reacted with the shed ectodomain of mouse COL17 but not with the full-length form (Figure 5, C-E). These results suggest that the Ab Mo-pyro-Glu preferentially targets neoepitopes on the shed mouse COL17 ectodomain, although Western blot using the highly concentrated $\mathrm{Ab}$ Mo-pyro-Glu and cell lysate from NMKs showed that the Ab was able to react weakly with the $180-\mathrm{kDa}$ full-length form (not shown).

To address the pathogenic roles of Abs targeting the neoepitopes on the shed COL17 ectodomain in blister formation, we performed passive transfer of the Ab Mo-pyroGlu and the Ab Mo-NC14A into neonatal and 8-week-old 
A
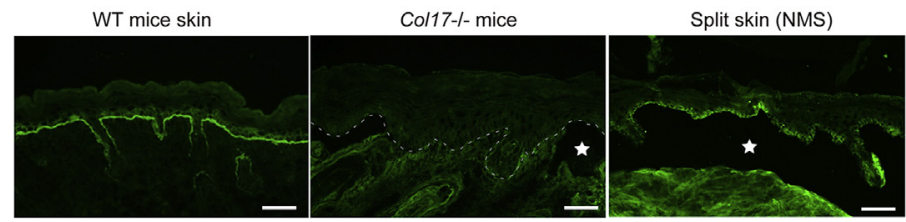

B

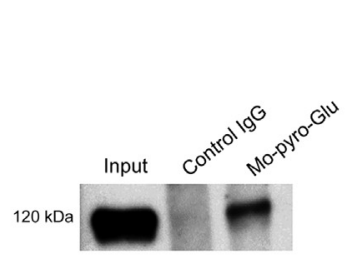

C
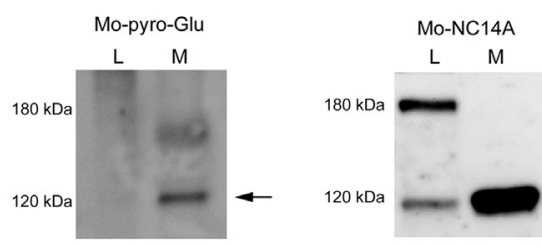

D

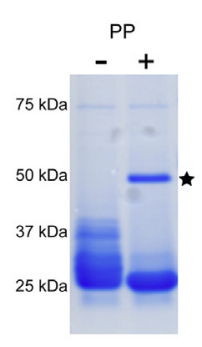

$\mathbf{F}$

Mo-pyro-Glu

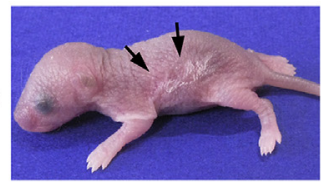

E
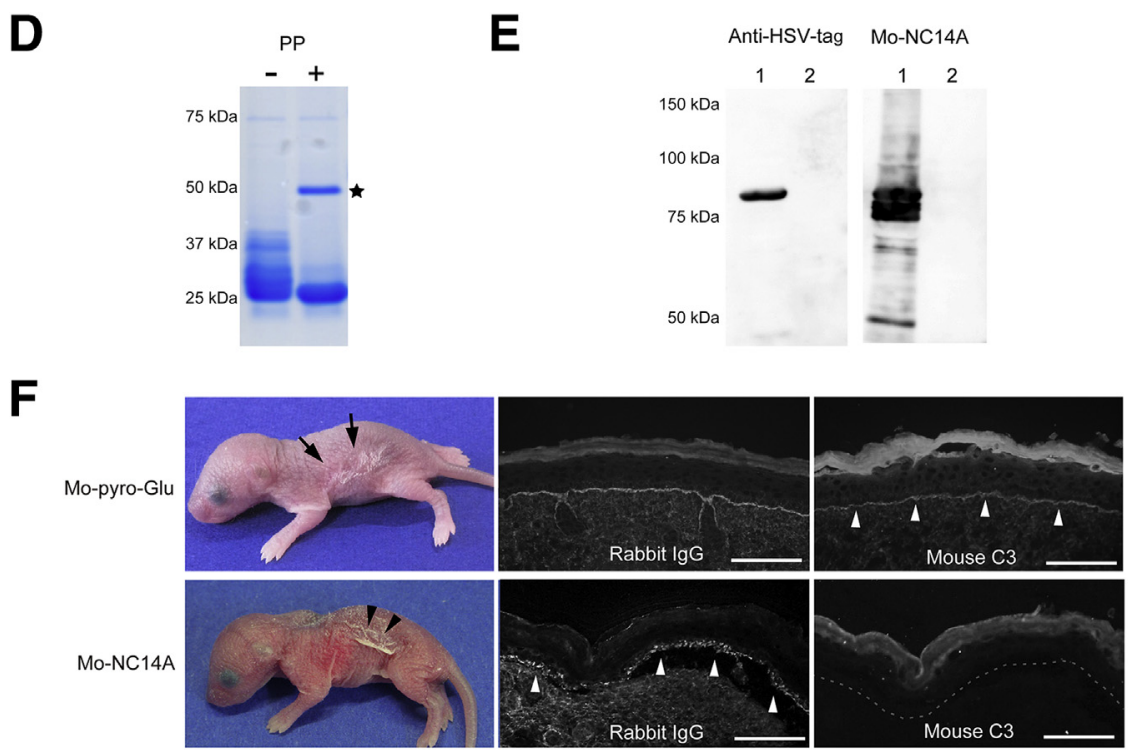

Normal rabbit lgG
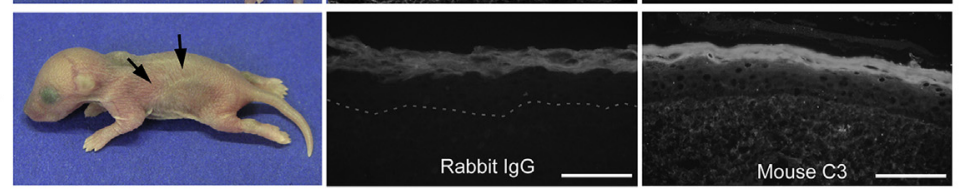

G
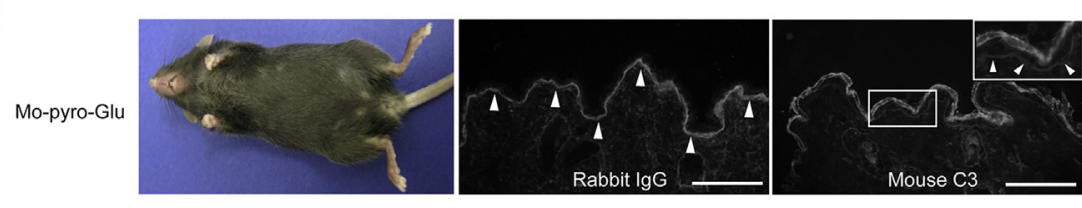

H

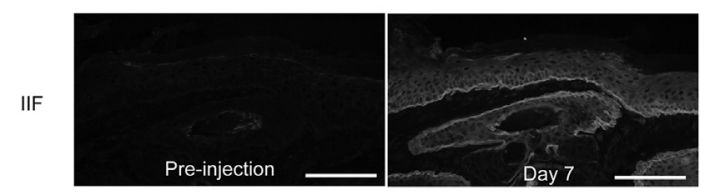

Figure 5 Pathogenic roles of Abs to neoepitopes on physiological cleavage sites of mouse collagen XVII (COL17). A: IIF using $2.25 \mathrm{ng} / \mu \mathrm{L}$ diluted affinitypurified antibody (Ab) Mo-pyro-Glu shows a strong reaction with the dermal-epidermal junction (DEJ) of the wild-type (WT) mouse skin, but it fails to react with COL17-lacking mouse $\left(\mathrm{Col}_{17^{-/}}\right)$skin. The Ab reacts with the epidermal side of $1 \mathrm{~mol} / \mathrm{L} \mathrm{NaCl}$ induced artificial blisters of normal mouse skin (NMS). Blisters are shown by stars. B: Immunoprecipitation of the shed COL17 ectodomain from normal mouse keratinocyte (NMK) culture medium by Mopyro-Glu. Ab Mo-pyro-Glu immunoprecipitates the $120-\mathrm{kDa}$ mouse COL17 ectodomain, which was detected by Ab Mo-NC14A. Input: concentrated medium protein. C: Western blot using cell lysate $(\mathrm{L})$ and culture medium (M) of NMKs. In contrast to the $A b$ Mo-NC14A reacting with both the full-length form and the shed ectodomain of mouse COL17, the Ab Mopyro-Glu only reacts with the shed 120-kDa ectodomain (arrow). The dim band around $150 \mathrm{kDa}$ is a nonspecific signal. D: The recombinant GST-NC14A protein is seen to migrate around $37 \mathrm{kDa}$ by Coomassie staining. Small fragments between 25 and 37 $\mathrm{kDa}$ are expected to be degraded or truncated proteins, which can be cleaved off by PreScission protease (star) (GE Healthcare). E: Western blot using recombinant NC14A-His.HSV protein (lane 1) and control protein from empty pET-44a vector (lane 2). Similar to anti-HSV-tag Abs, the Ab Mo-NC14A reacts with NC14A-His $\cdot H S V$, which migrates around $80 \mathrm{kDa}$. F: A neonatal C57BL/6 WT mouse 48 hours after receiving an intraperitoneal injection of $250 \mu \mathrm{g} / \mathrm{g}$ body weight affinity-purified Ab Mo-pyro-Glu. No epidermal detachment was observed (arrows), but direct immunofluorescence (DIF) studies show in vivo deposition of rabbit IgG and mouse C3 (white arrowheads) at the $D E J$ of skin from the injected mice. In contrast, a neonatal C57BL/6 WT mouse that received an intraperitoneal injection of $2000 \mu \mathrm{g} / \mathrm{g}$ body weight purified whole IgG containing Ab MoNC14A shows skin fragility (black arrowheads) associated with in vivo deposition of rabbit IgG (white arrowheads), but mouse C3 was not activated. No distinct abnormal findings were observed in the control mice that received normal rabbit IgG (arrows). G: An adult C57BL/6 mouse that received subcutaneous injection totaling $2500 \mu \mathrm{L}$ of antiserum containing the Ab Mo-pyro-Glu. No phenotypic abnormalities were observed. DIF studies showed in vivo deposition of rabbit IgG and mouse C3 (arrowheads) at the DEJ of the back skin of the injected mice. H: IIF studies using sera from adult WT mice that were injected with the Ab Mo-pyro-Glu. The treated mice, but not the control mice, had circulating Abs that strongly reacted with the DEJ of NMS at $400 \times$ dilution. Scale bars: $50 \mu \mathrm{m}(\mathbf{A}) ; 100 \mu \mathrm{m}(\mathbf{F}-\mathbf{H})$. HSV, herpes simplex virus; PP, PreScission protease.
C57BL/6J mice. Affinity-purified Ab Mo-pyro-Glu (10.72 $\mu \mathrm{g} / \mu \mathrm{L})$ with immunized peptides strongly reacted with the DEJ of the NMS, as observed by IIF (Figure 5A) at more than 1:32,000 dilution (not shown). Although the Ab Mo-pyroGlu in high concentration was able to react weakly with the
180-kDa full-length mouse COL17, we used the Abs for passive-transfer experiments without immunoadsorption because the treatment did not solely change the reactivity of the Ab Hu-pyro-Glu to the NHS, as mentioned earlier. As a result, high-dose passive transfer of the affinity-purified 
Ab Mo-pyro-Glu failed to induce skin fragility in any of the recipient mice by 48 hours after injection $(n=6)$ (Figure 5F), although direct immunofluorescence studies showed strong deposition of rabbit IgG and mouse $\mathrm{C} 3$ at the DEJ in all recipient mice (Figure 5F). In contrast, three fourths of neonatal mice that had received the $\mathrm{Ab}$ Mo-NC14A, which was affinity-purified by protein A (84.29 $\mu \mathrm{g} / \mu \mathrm{L}$ ), and reacts with NMS at more than 1:12,800 dilution, showed epidermal detachment by gentle mechanical friction (Figure 5F). Interestingly, direct immunofluorescence studies from the diseased mice showed in vivo deposits of rabbit IgG at the DEJ, although complement activation was very faint or largely absent (Figure 5F).

For adult mice, we performed the passive transfer of antiserum containing the Ab Mo-pyro-Glu, which reacts strongly with the DEJ of NMS as observed by IIF at more than 1:3200 dilution (not shown). Repeated passive transfer of antiserum containing the Ab Mo-pyro-Glu, totaling 2500 $\mu \mathrm{L}$ per mouse, did not induce any skin disease in recipient WT adult mice $(n=4)$, although in vivo deposition of rabbit IgG and mouse C3 was observed at the DEJ of the skin in all of the recipient mice (Figure 5G). In addition, the treated mice had circulating Abs that reacted with the DEJ of the NMS at more than $400 \times$ dilution (Figure 5H). Control mice that received the same dose of preimmune rabbit serum showed no phenotypical abnormalities, and no rabbit $\mathrm{IgG}$ or mouse $\mathrm{C} 3$ deposition was observed in the skin (not shown).

\section{Discussion}

COL17 is a key molecule for maintaining stable adhesion at the DEJ. Innate and acquired dysfunctions of the molecule, respectively, lead to the blistering disorders of non-Herlitz junctional epidermolysis bullosa ${ }^{10}$ and pemphigoid diseases. ${ }^{3}$ These blistering diseases suggest that COL17 plays a vital role in the stability of the skin. In contrast, here we showed that certain amounts of the COL17 ectodomain are cleaved physiologically even in steady-state normal human skin. COL17 is known to be expressed not only at the DEJ but also basolaterally and in the cytoplasm. ${ }^{11,15}$ However, the cleaved COL17 ectodomain detected by Abs targeting cleavage sites of the molecule (Ab Hu-pyro-Glu) was observed exclusively at the DEJ. This finding suggests that ectodomain shedding occurs only beneath the basal keratinocytes, probably within the hemidesmosomes. Similar findings have been reported for mAb 1337, another ectodomain-specific mAb reacting with bovine COL17. ${ }^{11}$

Interestingly, we found that a majority of N-terminal $\mathrm{Gln}^{525}$ of the shed COL17 ectodomain in NHEK culture medium was not cyclized into pyro-Glu. This finding was unexpected because, in a previous study, we identified pyro-Glu ${ }^{525}$ as a major N-terminal cleavage site by mass spectrometry analyses. ${ }^{15}$ The reason pyro-Glu was identified as a major Nterminal cleavage site probably is because N-terminal $\operatorname{Gln}^{525}$ spontaneously cyclized into pyro-Glu during repetitive sample preparation for mass spectrometry. Spontaneous N-terminal conversion from Glu to pyro-Glu is observed commonly in various proteins, including human $\mathrm{IgG} \mathrm{Abs} .{ }^{28}$ In contrast to in vitro culture systems, the present study showed that in vivo, the shed COL17 ectodomain in normal human skin has a cyclized pyro-Glu N-terminus, although it is uncertain whether the N-terminal cyclization of $\mathrm{Gln}^{525}$ occurs spontaneously or enzymatically in vivo.

It is well characterized that migrating activated keratinocytes express distinct adhesion molecules, such as integrin $\alpha 3 \beta 1$ and $\alpha 2 \beta 1$, at the basement membrane zone in vitro. ${ }^{29,30}$ In addition, wound keratinocytes are known to express these distinctive integrins. ${ }^{31}$ These findings suggest that different molecules are involved in keratinocyte migration. Previously, we showed that migrating keratinocytes cleave and leave the ectodomain of COL17 in the ECM as seen by the migration track in vitro, which was shown by Ab HK139, which targets the N-terminal cleavage site at $\mathrm{Leu}^{524} \cdot{ }^{15}$ This observation was associated somewhat with pathologic settings because we observed actively migrating keratinocytes. In contrast, here we showed that the $\mathrm{Ab}$ Hu-pyro-Glu did not react with the migration tracks of cultured NHEKs. Therefore, it is likely that the N-terminal cleavage of COL17 at $\operatorname{Gln}^{525}$ is associated with a physiological setting, rather than a pathologic one, which suggests that regulation of COL17 cleavage depends on the setting.

Alternatively, different cleavage sites on COL17 merely may be a result of different proteases involved under different settings, including normal steady phase or wound healing. However, what is the physiological function of COL17 ectodomain shedding in normal skin? It has been postulated that COL17 ectodomain shedding plays physiological roles associated with the migration, proliferation, and differentiation of basal keratinocytes. ${ }^{13}$ However, there has been no direct evidence for this. One possible physiological role may relate to the differentiation of basal keratinocytes because they must detach from the ECM to differentiate into the spinous and cornified layers. Alternatively, normal epidermis may be a more dynamic organ that communicates actively with ECM proteins through cell-surface receptors including COL17, whereby the ectodomain shedding of the molecule may be essential even in a normal setting.

The present study also suggests the presence of binding proteins of cleaved COL17 ectodomain in the ECM because IIF studies of cleavage site-specific Abs on $1 \mathrm{~mol} / \mathrm{L} \mathrm{NaCl}-$ split skin showed that the cleaved COL17 ectodomain remains on the epidermal side of artificial blisters. The C-terminus of COL17 binds with laminin 332 in vitro. ${ }^{24,32}$ Because laminin 332 always is present on the dermal side of $1 \mathrm{~mol} / \mathrm{L} \mathrm{NaCl}$-split skin,, 33 the present data indicate that another binding molecule(s) on the epidermal side is present in the ECM, which binds with the shed COL17 ectodomain more strongly than laminin 332. A candidate molecule is integrin $\alpha 6$, which is known to be able to bind with the 
ectodomain of COL17. ${ }^{34,35}$ Alternatively, additional cleavage may occur in the C-terminus of COL17 when artificial blisters form by $1 \mathrm{~mol} / \mathrm{L} \mathrm{NaCl}$. In any case, the present data suggest that the shed ectodomain of COL17 binds with another molecule(s) at the DEJ.

The ectodomain shedding of COL17 can evoke neoepitopes on the shed forms, which can be targeted by autoAbs of pemphigoid patients. ${ }^{16,17}$ Previously, we showed that linear B-cell epitopes have significantly increased antigenicity around cleavage sites within the NC16A domain, regardless of the physiological cleavage sites predicted in silico. ${ }^{15}$ However, the detailed pathomechanism of neoepitope generation has not been elucidated, and the pathologic role of autoAbs to neoepitopes still is uncertain in vivo. This study showed that modified N-terminal cleavage sites whose Gln has been changed into pyro-Glu produce distinct neoepitopes at the $\mathrm{N}$-terminal cleavage sites. We previously generated transgenic COL17-humanized mice for which the mouse COL17 was replaced by human COL17. The mice enabled us to reproduce blistering disease by passively transferring Abs to human COL17. ${ }^{9,21}$ Although the COL17-humanized mouse skin expresses full-length human COL17, the Ab Hu-pyro-Glu did not stain at the DEJ. Similarly, the cleavage site-specific $\mathrm{Ab}$ HK139 targeting N-terminal Leu ${ }^{524}$ did not react with the COL17-humanized mouse skin (not shown, unpublished data). It still is uncertain why these Abs lost their reactivity to human COL17 in the transgenic mice. One possible mechanism is that the cleavage of human COL17 in the COL17-humanized transgenic mouse skin may be different from that in NHS.

In this study, we produced rabbit Abs targeting a candidate cleavage site of the mouse COL17 ectodomain (Ab Mo-pyro-Glu), which preferentially react with the shed mouse COL17. The passive transfer of the Ab Mo-pyro-Glu failed to induce blistering diseases in neonatal and adult WT mice, even though injected rabbit IgG bound with mouse COL17 and activated mouse C 3 in vivo. Previous studies have shown that in vivo deposition of Abs to the NC16A domain (the NC14A domain for mouse COL17) of COL17 associated with complement activation is a hallmark of neonatal BP models, ${ }^{8,36}$ although we recently found that complement activation is not necessary for blistering diseases in mice. ${ }^{21,22,37}$ In any case, we found no evidence that Abs targeting a candidate physiological $\mathrm{N}$-terminal cleavage site are pathogenic in vivo. However, epitopes can be changed significantly in regions far from the cleavage sites. For example, the mAb NC16A-3, whose epitope is located at least 19 amino acids C-terminus from the physiological cleavage site within the NC16A domain of COL17, reacts more strongly with the shed ectodomain than with the fulllength form. ${ }^{20}$ In addition, the 15 th collagenous domain is known to be a candidate epitope for autoAbs from linear IgA bullous dermatosis patients, ${ }^{38}$ which suggests that the conformation of the COL17 ectodomain may change drastically after cleavage within the NC16A domain. Therefore, our study does not rule out the possibility that Abs reacting with other neoepitopes far from the physiological cleavage sites may be pathogenic for blister formation.

In summary, we showed that the ectodomain of COL17 is cleaved physiologically in vivo in human skin, which produces distinct neoepitopes at the cleavage sites with pyroglutamate $\mathrm{N}$-termini. Although rabbit Abs to the shed ectodomain bind with mouse COL17 and activate murine complements in vivo, blistering disease is not introduced, suggesting that an immune reaction to the neoepitopes on the physiological N-terminal cleavage sites would not be pathogenic.

\section{Acknowledgments}

We thank Maiko Tozawa for technical assistance. The rabbit polyclonal $\mathrm{Abs}$ and mouse mAb NC16A-3 were provided by Prof. Bruckner-Tuderman, (Freiburg Institute for Advanced Studies, Freiburg im Breisgau, BadenWürttemberg, Germany).

\section{References}

1. Diaz LA, Ratrie H 3rd, Saunders WS, Futamura S, Squiquera HL Anhalt GJ, Giudice GJ: Isolation of a human epidermal cDNA corresponding to the $180-\mathrm{kD}$ autoantigen recognized by bullous pemphigoid and herpes gestationis sera. Immunolocalization of this protein to the hemidesmosome. J Clin Invest 1990, 86:1088-1094

2. Stanley JR: Pemphigus and pemphigoid as paradigms of organspecific, autoantibody-mediated diseases. J Clin Invest 1989, 83: $1443-1448$

3. Schmidt E, Zillikens D: Pemphigoid diseases. Lancet 2013, 381: $320-332$

4. Ishiko A, Shimizu H, Kikuchi A, Ebihara T, Hashimoto T, Nishikawa T: Human autoantibodies against the $230-\mathrm{kD}$ bullous pemphigoid antigen (BPAG1) bind only to the intracellular domain of the hemidesmosome, whereas those against the $180-\mathrm{kD}$ bullous pemphigoid antigen (BPAG2) bind along the plasma membrane of the hemidesmosome in normal human and swine skin. J Clin Invest 1993 , 91:1608-1615

5. Nishie W: Update on the pathogenesis of bullous pemphigoid: an autoantibody-mediated blistering disease targeting collagen XVII. J Dermatol Sci 2014, 73:179-186

6. Zillikens D, Rose PA, Balding SD, Liu Z, Olague-Marchan M, Diaz LA, Giudice GJ: Tight clustering of extracellular BP180 epitopes recognized by bullous pemphigoid autoantibodies. J Invest Dermatol 1997, 109:573-579

7. Kobayashi M, Amagai M, Kuroda-Kinoshita K, Hashimoto T, Shirakata Y, Hashimoto K, Nishikawa T: BP180 ELISA using bacterial recombinant $\mathrm{NC16}$ a protein as a diagnostic and monitoring tool for bullous pemphigoid. J Dermatol Sci 2002, 30:224-232

8. Liu Z, Diaz LA, Troy JL, Taylor AF, Emery DJ, Fairley JA, Giudice GJ: A passive transfer model of the organ-specific autoimmune disease, bullous pemphigoid, using antibodies generated against the hemidesmosomal antigen, BP180. J Clin Invest 1993, 92: 2480-2488

9. Nishie W, Sawamura D, Goto M, Ito K, Shibaki A, McMillan JR, Sakai K, Nakamura H, Olasz E, Yancey KB, Akiyama M, Shimizu H: Humanization of autoantigen. Nat Med 2007, 13:378-383

10. Gatalica B, Pulkkinen L, Li K, Kuokkanen K, Ryynanen M, McGrath JA, Uitto J: Cloning of the human type XVII collagen gene (COL17A1), and detection of novel mutations in generalized atrophic benign epidermolysis bullosa. Am J Hum Genet 1997, 60:352-365 
11. Hirako Y, Usukura J, Uematsu J, Hashimoto T, Kitajima Y, Owaribe K: Cleavage of BP180, a 180-kDa bullous pemphigoid antigen, yields a 120-kDa collagenous extracellular polypeptide. J Biol Chem 1998, 273:9711-9717

12. Franzke CW, Tasanen K, Schacke H, Zhou Z, Tryggvason K, Mauch C, Zigrino P, Sunnarborg S, Lee DC, Fahrenholz F, BrucknerTuderman L: Transmembrane collagen XVII, an epithelial adhesion protein, is shed from the cell surface by ADAMs. EMBO J 2002, 21: 5026-5035

13. Franzke CW, Tasanen K, Schumann H, Bruckner-Tuderman L: Collagenous transmembrane proteins: collagen XVII as a prototype. Matrix Biol 2003, 22:299-309

14. Hirako Y, Nishizawa Y, Sitaru C, Opitz A, Marcus K, Meyer HE, Butt E, Owaribe K, Zillikens D: The 97-kDa (LABD97) and 120-kDa (LAD-1) fragments of bullous pemphigoid antigen 180/type XVII collagen have different N-termini. J Invest Dermatol 2003, 121:1554-1556

15. Nishie W, Lamer S, Schlosser A, Licarete E, Franzke CW, Hofmann SC, Jackow J, Sitaru C, Bruckner-Tuderman L: Ectodomain shedding generates neoepitopes on collagen XVII, the major autoantigen for bullous pemphigoid. J Immunol 2010, 185:4938-4947

16. Zillikens D, Herzele K, Georgi M, Schmidt E, Chimanovitch I, Schumann H, Mascaro JM Jr, Diaz LA, Bruckner-Tuderman L, Brocker EB, Giudice GJ: Autoantibodies in a subgroup of patients with linear IgA disease react with the NC16A domain of BP1801. J Invest Dermatol 1999, 113:947-953

17. Schumann H, Baetge J, Tasanen K, Wojnarowska F, Schacke H, Zillikens D, Bruckner-Tuderman L: The shed ectodomain of collagen XVII/BP180 is targeted by autoantibodies in different blistering skin diseases. Am J Pathol 2000, 156:685-695

18. Marinkovich MP, Taylor TB, Keene DR, Burgeson RE, Zone JJ: LAD-1, the linear IgA bullous dermatosis autoantigen, is a novel 120$\mathrm{kDa}$ anchoring filament protein synthesized by epidermal cells. J Invest Dermatol 1996, 106:734-738

19. Zone JJ, Taylor TB, Meyer LJ, Petersen MJ: The $97 \mathrm{kDa}$ linear IgA bullous disease antigen is identical to a portion of the extracellular domain of the $180 \mathrm{kDa}$ bullous pemphigoid antigen, BPAg2. J Invest Dermatol 1998, 110:207-210

20. Hofmann SC, Voith U, Schonau V, Sorokin L, Bruckner-Tuderman L, Franzke CW: Plasmin plays a role in the in vitro generation of the linear IgA dermatosis antigen LADB97. J Invest Dermatol 2009, 129:1730-1739

21. Natsuga K, Nishie W, Shinkuma S, Ujiie H, Nishimura M, Sawamura D, Shimizu H: Antibodies to pathogenic epitopes on type XVII collagen cause skin fragility in a complement-dependent and -independent manner. J Immunol 2012, 188:5792-5799

22. Ujiie H, Sasaoka T, Izumi K, Nishie W, Shinkuma S, Natsuga K, Nakamura H, Shibaki A, Shimizu H: Bullous pemphigoid autoantibodies directly induce blister formation without complement activation. J Immunol 2014, 193:4415-4428

23. Langhofer M, Hopkinson SB, Jones JC: The matrix secreted by $804 \mathrm{G}$ cells contains laminin-related components that participate in hemidesmosome assembly in vitro. J Cell Sci 1993, 105:753-764

24. Nishie W, Kiritsi D, Nystrom A, Hofmann SC, Bruckner-Tuderman L: Dynamic interactions of epidermal collagen XVII with the extracellular matrix: laminin 332 as a major binding partner. Am J Pathol 2011, $179: 829-837$

25. Nakamura H, Sawamura D, Goto M, Kida M, Ariga T, Sakiyama Y, Tomizawa K, Mitsui H, Tamaki K, Shimizu H: Analysis of the COL17A1 in non-Herlitz junctional epidermolysis bullosa and amelogenesis imperfecta. Int J Mol Med 2006, 18:333-337

26. Li K, Tamai K, Tan EM, Uitto J: Cloning of type XVII collagen. Complementary and genomic DNA sequences of mouse 180kilodalton bullous pemphigoid antigen (BPAG2) predict an interrupted collagenous domain, a transmembrane segment, and unusual features in the $5^{\prime}$-end of the gene and the $3^{\prime}$-untranslated region of the mRNA. J Biol Chem 1993, 268:8825-8834

27. Xu W, Peng Y, Wang F, Paporello B, Richardson D, Liu H: Method to convert N-terminal glutamine to pyroglutamate for characterization of recombinant monoclonal antibodies. Anal Biochem 2013, 436:10-12

28. Liu YD, Goetze AM, Bass RB, Flynn GC: N-terminal glutamate to pyroglutamate conversion in vivo for human $\operatorname{IgG} 2$ antibodies. J Biol Chem 2011, 286:11211-11217

29. Carter WG, Wayner EA, Bouchard TS, Kaur P: The role of integrins alpha 2 beta 1 and alpha 3 beta 1 in cell-cell and cell-substrate adhesion of human epidermal cells. J Cell Biol 1990, 110:1387-1404

30. Tsuruta D, Hashimoto T, Hamill KJ, Jones JC: Hemidesmosomes and focal contact proteins: functions and cross-talk in keratinocytes, bullous diseases and wound healing. J Dermatol Sci 2011, 62:1-7

31. Larjava H, Salo T, Haapasalmi K, Kramer RH, Heino J: Expression of integrins and basement membrane components by wound keratinocytes. J Clin Invest 1993, 92:1425-1435

32. Tasanen K, Tunggal L, Chometon G, Bruckner-Tuderman L, Aumailley M: Keratinocytes from patients lacking collagen XVII display a migratory phenotype. Am J Pathol 2004, 164:2027-2038

33. Shimizu $H$, Masunaga $T$, Ishiko A, Matsumura $K$, Hashimoto $T$, Nishikawa T, Domloge-Hultsch N, Lazarova Z, Yancey KB: Autoantibodies from patients with cicatricial pemphigoid target different sites in epidermal basement membrane. J Invest Dermatol 1995, 104:370-373

34. Hopkinson SB, Baker SE, Jones JC: Molecular genetic studies of a human epidermal autoantigen (the 180-kD bullous pemphigoid antigen/BP180): identification of functionally important sequences within the BP180 molecule and evidence for an interaction between BP180 and alpha 6 integrin. J Cell Biol 1995, 130:117-125

35. Hopkinson SB, Findlay K, deHart GW, Jones JC: Interaction of BP180 (type XVII collagen) and alpha6 integrin is necessary for stabilization of hemidesmosome structure. J Invest Dermatol 1998, 111:1015-1022

36. Liu Z, Giudice GJ, Swartz SJ, Fairley JA, Till GO, Troy JL, Diaz LA: The role of complement in experimental bullous pemphigoid. J Clin Invest 1995, 95:1539-1544

37. Nishie W, Sawamura D, Natsuga K, Shinkuma S, Goto M, Shibaki A, Ujiie H, Olasz E, Yancey KB, Shimizu H: A novel humanized neonatal autoimmune blistering skin disease model induced by maternally transferred antibodies. J Immunol 2009, 183:4088-4093

38. Nie Z, Nagata Y, Joubeh S, Hirako Y, Owaribe K, Kitajima Y, Hashimoto T: IgA antibodies of linear IgA bullous dermatosis recognize the 15th collagenous domain of BP180. J Invest Dermatol 2000, 115:1164-1166 